Variant of Nov 2000. Last reviews July/Sept 2001.

\title{
Elementary equivalence versus Isomorphism
}

\author{
by Florian Pop at Bonn
}

\section{Introduction}

The aim of this note is to give some new evidence for a long standing open question concerning the relation between elementary equivalence and isomorphism in the class of finitely generated fields. More precisely, we will give a positive answer to this question in the "general" case.

We recall that two fields $K$ and $L$ are called elementarily equivalent, if for every sentence $\psi$ in the language of fields on one has: $\psi$ is true in $K$ if and only if $\psi$ is true in $L$. Just to start with, we recall that the assertion " $K$ admits an ordering" can be expressed in an elementary way (by a scheme of axioms) by saying that " -1 is not a sum of squares in $K$ ", wheres the fact that a function field $K \mid \mathbb{Q}$ has transcendence degree $d$ cannot be expressed in an obvious way (or not at all), as we would have to say that "there exist $t_{1}, \ldots, t_{d} \in K$ such that for all non-zero polynomials $p\left(X_{1}, \ldots, X_{d}\right)$ over $\mathbb{Q}$ one has $p\left(t_{1}, \ldots, t_{d}\right) \neq 0$, and every other element of $K$ is algebraic over $\mathbb{Q}\left(t_{1}, \ldots, t_{d}\right)$ ". Clearly, this is not elementarily expressable - at least notin an obvious way. Concerning this we make the following remarks:

1) For any field $K$ let $\mathfrak{T h}(K)$ be the set of sentences in the language of fields, which are true in $K$. Then $K$ and $L$ are elementarily equivalent if and only if $\mathfrak{T h}(K)=\mathfrak{T h}(L)$. Clearly, if $K \cong L$ as fields, then $\mathfrak{T h}(K)=\mathfrak{T h}(L)$, thus $K$ and $L$ are elementarily equivalent.

2) The precise relation between elementary equivalence and isomorphism is as follows: $K$ and $L$ are elementarily equivalent iff there exit ultra-powers $K^{*}=K^{I} / \mathcal{D}$ and $L^{*}=L^{J} / \mathcal{E}$ which are isomorphic as fields. In particular, if $K$ and $L$ are elementarily equivalent, then they have the same prime field $k$, and if $K^{\text {abs }}$ and $L^{\text {abs }}$ denote their "absolute subfields" (i.e., the relative algebraic closures of $k$ ) in $K$ and in $L$ respectively, then $K^{\text {abs }} \cong L^{\text {abs }}$.

3) From a more geometric point of view, one can express the elementary equivalence as follows: $K$ and $L$ are elementarily equivalent if and only if 
they have the same prime field $k$, and for every constructible subset $S$ of a scheme of finite type over $k$ one has: $S(K)$ non-empty if and only if $S(L)$ non-empty.

It appears to be an interesting and intriguing question, whether for finitely generated fields, the elementary equivalence if the same as the isomorphism. The first non-obvious question in this direction is whether the absolute transcendence degree of a finitely generated field can be described by an assertion in the language of fields. It seems that the following more precise question was asked by SABBAGH in the beginning if the Eighties: Let $K$ be a function field of one variable over $\mathbb{Q}$, and $L$ the rational function field in two variables over $\mathbb{Q}$. Is it then possible that $K$ and $L$ are elementarily equivalent? Part of the problem here is clearly the question, whether one could find assertions in the language of fields which can distinguish between the transcendence degrees 1 and 2 of function fields over $\mathbb{Q}$. Before giving the main results, let us recall that a function field $K \mid \kappa$ over some base field $\kappa$ is said to be of general type, if it is the function field of a projective smooth variety of general type $X \rightarrow \kappa$. Correspondingly, we say that a finitely generated field $K$ is of general type, if it is of general type over its absolute subfield $\kappa=K^{\text {abs }}$. The main results of this note are the following:

Theorem A (Arithmetic variant). Let $K$ and $L$ be finitely generated fields which are elementarily equivalent. Let $\kappa$ and $\lambda$ be their absolute subfields. Then one has:

(1) $\kappa$ and $\lambda$ are isomorphic, and $\operatorname{td}(K \mid \kappa)$ equals $\operatorname{td}(L \mid \lambda)$.

(2) Moreover, there exits an embedding $\imath: K \rightarrow L$ such that $L$ is finite separable over $\imath(K)$. Furthermore, if $K$ is of general type, then $K \cong L$ as fields.

Theorem B (Geometric variant). Let $K \mid \kappa$ and $L \mid \lambda$ be function fields over algebraically closed fields $\kappa$, respectively $\lambda$. Suppose that $K$ and $L$ are elementarily equivalent as fields. The one has:

(1) $\kappa$ and $\lambda$ are elementarily equivalent, and $\operatorname{td}(K \mid \kappa)$ equals $\operatorname{td}(L \mid \lambda)$.

(2) Suppose $K \mid \kappa$ is of general type. Then there exist function subfields $K_{0}\left|\kappa_{0} \hookrightarrow K\right| \kappa$ and $L_{0}\left|\lambda_{0} \hookrightarrow L\right| \lambda$ such that $K=K_{0} \kappa$ and $L=L_{0} \lambda$, and $K_{0}\left|\kappa_{0} \cong L_{0}\right| \lambda_{0}$ as function fields.

In particular, if $\kappa \cong \lambda$ are isomorphic, then $K|\kappa \cong L| \lambda$ are isomorphic as function fields. 
We would like to mention that there are already results in the literature concerning the geometric case by DURET [D1], [D2], and PIERCE [Pi], which completely answer the case of function fields of curves, thus working under the hypothesis $\operatorname{td}(K \mid \kappa)=1=\operatorname{td}(L \mid \lambda)$. Among other things they show that the result is true also for the function fields of elliptic curves without complex multiplication. This goes beyond Theorem B in the case under discussion, as the elliptic curves are not curves of general type.

It remains an open question to give the precise relation between elementary equivalence and isomorphism in the "non-general" case.

We would also like to say that a preliminary form of the above results, precisely: the characterisation of the transcendence degree by Pfister forms was known to the author already in 1998; and Theorem A in the case $\operatorname{td}(K \mid \kappa) \leq 1$, but the approach was different. The main tool was the Mordell Conjecture (as proved by FALTINGS). In particular, we exploited the relation between rational points on general curves and the elementary theory of function fields of curves over number fields. It remains a serious open question to precisely understand in general the relation between these two apparently different problems.

\section{Section 1. Detecting the transcendence degree}

In this subsection we show how to detect the transcendence degree -in the cases under discussion- by "nice" sentences in the language of fields. In the positive characteristic case and in the geometric case, i.e., if the base field is algebraically closed, the answer to this question is "easy", and might well already be known. Therefore we will only indicate how to get it. Nevertheless, in the arithmetic case, i.e., in the case of finitely generated fields over number fields, the only way we can do this is by using the the Milnor Conjecture, proved by Voevodsky, Rost, ET AL, see KAHN's [Kh] talk in Séminaire Bourbaki.

We consider the context: $K \mid \kappa$ is a function field with $\operatorname{td}(K \mid \kappa)=d>0$.

A) $\kappa$ has positive characteristic

Suppose $\kappa$ is a perfect field of characteristic $p>0$, in particular $\kappa$ might be finite or algebraically closed. Thus, if $K^{p}$ denotes the subfield of $K$ consisting of all the $p$-powers in $K$, then $K \mid K^{p}$ is a (finite) purely inseparable 
extension. Since $\kappa$ is a perfect field, it follows that $\left[K: K^{p}\right]=p^{d}$, where $d$ is the transcendence degree of $K \mid \kappa$. This fact can be easily expressed by a sentence in the language of fields. But a more precise formulation exists, by which we can say that a given system of elements of $K$ is a separable transcendence basis of $K \mid \kappa$ as follows. We recall the following two definitions: (1) First, a system of elements $\mathcal{B}_{r}=\left(t_{1}, \ldots, t_{r}\right)$ of $K$ is said to be $p$-independent, if the system of all the monomials $\underline{t} \underline{i}$ of the following form $\underline{t}-t_{1}^{i} \ldots t_{d}^{i_{r}}, 0 \leq i_{1}, \ldots, i_{r}<p$, is linearly independent over $K^{p}$. (2) Second, a system of elements $\mathcal{B}_{d}=\left(t_{1}, \ldots, t_{d}\right)$ of $K$ is said to be a $p$-basis, if the set of all the monomials as above is a vector space basis of $K$ over $K^{p}$.

It is clear that both assertion (1) and assertion (2) can be interpreted as formulas in the language of fields with no parameters excepting $t_{1}, \ldots, t_{r}$. Namely for a given system $\mathcal{B}_{r}=\left(t_{1}, \ldots, t_{r}\right)$ of elements of $K$, let $\underline{i}=$ $\left(i_{1}, \ldots, i_{r}\right)$ be all the multi-indexes with $0 \leq i_{j}<p$, and define the following form of degree $p$ in the $p^{r}$ variables $X_{\underline{i}}$ over $K$ :

$$
q_{\mathcal{B}_{r}}^{(p)}\left(X_{1}, \ldots, X_{p^{r}}\right)=\sum_{\underline{i}} \underline{\underline{t}} \underline{\underline{i}} X_{\underline{i}}^{p}
$$

Then the form $q_{\mathcal{B}_{r}}^{(p)}$ does not represent 0 over $K$ if and only if $\mathcal{B}_{r}$ is $p$ independent; and the form $q_{\mathcal{B}_{d}}^{(p)}$ is universal over $K$, but does not represent 0 over $K$ if and only if $\mathcal{B}_{d}$ is a $p$-basis of $K \mid \kappa$.

The relation of this with the transcendence bases of $K \mid \kappa$ is given by the following well known fact, see e.g. [Ei], Appendix 1.

Fact 1.1. Let $K \mid \kappa$ be a function field with $\kappa$ is a perfect field of characteristic $p>0$. For a system of elements $\mathcal{B}_{r}=\left(t_{1}, \ldots, t_{r}\right)$ of $K$ the following assertions hold:

(1) $\mathcal{B}_{r}$ is p-independent $\Leftrightarrow \mathcal{B}_{r}$ can be completed to a separable transcendence basis of $K \mid \kappa \Leftrightarrow q_{\mathcal{B}_{r}}^{(p)}$ does not represent 0 over $K$.

(2) $\mathcal{B}_{d}$ is a p-basis $\Leftrightarrow \mathcal{B}_{d}$ is a separable transcendence basis of $K \mid \kappa \Leftrightarrow$ the form $q_{\mathcal{B}_{d}}^{(p)}$ is universal over $K$, but does not represent 0 over $K$.

(3) In particular, $K \mid \kappa$ has transcendence degree $d$ if and only if the following sentence is true in $K$ : There exists $\mathcal{B}_{d}=\left(t_{1}, \ldots, t_{d}\right)$ such that $q_{\mathcal{B}_{d}}^{(p)}$ is universal over $K$, but does not represent 0 over $K$. 
B) $\kappa$ is algebraically closed

Let $K \mid \kappa$ be an a function field with $\kappa$ an algebraically closed field of characteristic $\neq 2$. It is interesting to remark that in the case $p=2$, the form $q_{\mathcal{B}_{r}}:=q_{\mathcal{B}_{r}}^{(2)}$ defined above is a very special quadratic form, namely the $r$-fold Pfister form attached to the system of elements $\mathcal{B}_{r}$ of $K$. On the other hand, since $K \mid \kappa$ is a function field of transcendence degree $d$, and $\kappa$ is algebraically closed, $K$ is a $C_{d}$ field. For quadratic forms, this means that every such form in $n>2^{d}$ variables represents 0 over $K$. In particular, if $\operatorname{char}(\kappa) \neq 2$, every $d$-fold Pfister form $q=q_{\mathcal{B}}$ is universal. (In the case char $(\kappa)=2$ we know already what happens from the previous subsection.) The point is that in this way we can characterize the transcendence degree of $K \mid \kappa$, and even more, determine whether a system of elements $\mathcal{B}_{d}=\left(t_{1}, \ldots, t_{d}\right)$ of $K$ is a transcendence basis of $K \mid \kappa$.

Fact 1.2. Let $K \mid \kappa$ be a function field with $\kappa$ an algebraically closed field with $\operatorname{char}(\kappa) \neq 2$. To systems $\mathcal{B}_{r}=\left(t_{1}, \ldots, t_{r}\right)$ of elements $K$ we denote by $q_{\mathcal{B}_{r}}$ the corresponding Pfister form. Then the following holds:

(1) $K \mid \kappa$ has transcendence degree $d \Leftrightarrow$ for all $\mathcal{B}_{d}$ the corresponding $q_{\mathcal{B}_{d}}$ is universal over $K$, and there exit $\mathcal{B}_{d}$ such that $q_{\mathcal{B}_{d}}$ does not represent 0 over $K$.

(2) Suppose that $\operatorname{td}(K \mid \kappa)=d$. For a system of elements $\mathcal{B}_{d}$ of $K$ suppose that $q_{\mathcal{B}_{d}}$ is universal, but does not represent 0 over $K$. Then $\mathcal{B}_{d}$ is a transcendence basis of $K \mid \kappa$.

(3) In particular, let $\mathcal{S} \subset \kappa$ be an infinite subset, and for a systems $\mathcal{B}_{r}$ as above, and $a=\left(a_{1}, \ldots, a_{r}\right)$ set $\mathcal{B}_{r, a}=\left(t_{1}-a_{1}, \ldots, t_{r}-a_{r}\right)$. Then $\mathcal{B}_{r}$ is a transcendence base for $K \mid \kappa$ if and only if for "almost all" a the corresponding form $q_{\mathcal{B}_{r, a}}$ is universal, but does not represent 0 over $K$.

Proof. First, since $\kappa$ is algebraically closed, $K$ is a $C_{d}$ field. Thus, for every $a \in K^{\times}$, the quadratic form $q=a X^{2}-q_{\mathcal{B}_{d}}$ has $2^{d}+1$ variables, hence it represents 0 over $K$. Since $\operatorname{char}(\kappa) \neq 2$, from this is follows that $q_{\mathcal{B}_{d}}$ represents $a$ over $K$. The remaining assertions can be derived as follows. Let $X \rightarrow \kappa$ be some model of $K \mid \kappa$, and let $x \in X$ be a closed regular (thus smooth) point. Then for every regular system of parameters $\left(t_{1}, \ldots, t_{d}\right)$ at $x$ one has the following

Claim: The quadratic form $q_{\left(t_{1}, \ldots, t_{d}\right)}$ does not represent 0 over $K$. 
Indeed, it is a well known fact that, since $x$ is a regular closed point, $K$ has $\kappa$-embeddings in $\Lambda_{d}=\kappa\left(\left(t_{1}\right)\right) \ldots\left(\left(t_{d}\right)\right)$. One shows now by induction on $r$, that the $r$-fold Pfister form $q_{\left(t_{1}, \ldots, t_{r}\right)}=\left\langle<t_{1}, \ldots, t_{r}>>\right.$ is universal over $\Lambda_{r}$, but does not represent 0 . Finally, for the last assertion, remark that $r \leq \operatorname{td}(K \mid \kappa)$, as the form $q_{\mathcal{B}_{r, a}}$ does not represent 0 over $K$. If now $r<\operatorname{td}(K \mid \kappa)$, then for a proper choice of a closed point $x$ as above, after setting $a_{i}=t_{i}(x)$, it follows that $\left(t_{1}-a_{1}, \ldots, t_{r}-a_{r}\right)$ is contained in a regular system of parameters at $x$. Thus if $r<\operatorname{td}(K \mid \kappa)$, and $\left(t_{r+1}, \ldots, t_{d}\right)$ are the extra-parameters, then none of these is represented by $q_{\mathcal{B}_{r, a}}$ over $\Lambda_{d, a}=\kappa\left(\left(t_{1}-a_{1},\right)\right) \ldots\left(\left(t_{d}\right)\right)$, thus nor over $K$.

C) $\kappa$ is a number field

We now come to the discussion of the arithmetic case, which is the most interesting (but the more difficult) one. Thus here $K \mid \kappa$ is a function field over a number field $\kappa$. As above, to systems $\mathcal{B}_{r}=\left(t_{1}, \ldots, t_{r}\right)$ of elements of $K$, we denote by $q_{\mathcal{B}_{r}}$ the corresponding Pfister form.

Fact 1.3. Let $K \mid \kappa$ be a function field with $\kappa$ a number field. Then the following holds:

(1) $K \mid \kappa$ has transcendence degree $d \Leftrightarrow$ for all $\mathcal{B}_{d+2}$ the corresponding quadratic form $q_{\mathcal{B}_{d+2}}$ is universal over $K[\sqrt{-1}]$, and there exits $\mathcal{B}_{d+2}$ such that $q_{\mathcal{B}_{d+1}}$ does not represent 0 over $K[\sqrt{-1}]$.

(2) Suppose that $\operatorname{td}(K \mid \kappa)=d$. For a system of elements $\mathcal{B}_{d}=\left(t_{1}, \ldots, t_{d}\right)$ of $K$ suppose that there exit $t_{d+1}, t_{d+2} \in \kappa$ such that $q_{\left(t_{1}, \ldots, t_{d+2}\right)}$ does not represent 0 over $K[\sqrt{-1}]$. Then $\mathcal{B}_{d}$ is a transcendence basis of $K \mid \kappa$.

Proof. We proof goes along the following steps.

\section{a) Some cohomological computations}

For a field extension $K \mid \kappa$ as above, and a prime number $\ell$, we denote by $\operatorname{vcd}_{\ell}(K)$ the virtual $\ell$-cohomological dimension of $K$. It actually equals $\operatorname{cd}_{\ell}(E)$ for every non-real finite extension $E \mid K$. In particular, if $\sqrt{-1}$ lies in the field in discussion, then $\operatorname{vcd}_{\ell}=\mathrm{cd}_{\ell}$ for every $\ell$.

By classical results in Galois cohomology of fields, see e.g. SERRE [S], Ch.II, one has: First, if $\ell=\operatorname{char}(K)$, then $\operatorname{cd}_{\ell}(K)=1$ (this is a theorem of Shafarevich). Second, if $K$ is finite, then $\operatorname{cd}_{\ell}(K)=1$ for all $\ell$, and if $K$ is a number field, then $\operatorname{vcd}_{\ell}(K)=2$ for all $\ell$ (this is a theorem of Tate). 
Combined with the fact that $\operatorname{vcd}_{\ell}(\kappa(t))=\operatorname{vcd}_{\ell}(\kappa)+1$ for all fields $\kappa$ and $\ell \neq \operatorname{char}(\kappa)$, we get:

Fact. In the context of Fact 1.3 one has: $\operatorname{td}(K \mid \kappa)=d=\operatorname{vcd}_{\ell}(K)-2$ for all rational prime numbers $\ell$.

Let $\mu_{\ell}$ be the group of $\ell^{\text {th }}$ roots of unity. Then by Kummer theory and the tame symbol from the Milnor $K$-theory, we have a canonical isomorphism $\delta: K^{\times} / \ell \cong \mathrm{H}^{1}\left(G_{K}, \mu_{\ell}\right)$, which induces canonical homomorphisms

$$
h: K^{\times} \otimes \ldots \otimes K^{\times} \rightarrow \mathrm{K}_{n}^{\mathrm{M}}(K) / \ell \stackrel{d}{\longrightarrow} \mathrm{H}^{n}\left(G_{K}, \mu_{\ell}^{\otimes n}\right),
$$

denoted $\left(a_{1}, \ldots, a_{n}\right) \mapsto\left\{a_{1}, \ldots, a_{n}\right\} \mapsto a_{1} \cup \ldots \cup a_{n}$, see e.g. Milnor [M1]. We further remark, that if $\mu_{\ell} \subset K$, then $\mathrm{H}^{1}\left(G_{K}, \mu_{\ell}\right) \cong \operatorname{Hom}\left(G_{K}, \mathbb{Z} / \ell\right)$ is the character group of $G_{K}$ with values in $\mathbb{Z} / \ell$, and second, $\mathrm{H}^{d}\left(G_{K}, \mu_{\ell}^{\otimes n}\right)$ can be identified with $\mathrm{H}^{n}\left(G_{K}, \mathbb{Z} / \ell\right)$. The main observation is now the following

Lemma. In the context of Fact 1.3, let $\ell$ be a prime number. Let $E$ be a finite extension of $K$ containing $\mu_{2 \ell}$. Then one can detect $m=\operatorname{vcd}_{\ell}(K)$ as the unique natural number $m$ with the following properties:

(i) There exist $a_{1}, \ldots, a_{m}$ in $K^{\times}$such that $a_{1} \cup \ldots \cup a_{m} \neq 0$ as element of $\mathrm{H}^{m}\left(G_{E}, \mathbb{Z} / \ell\right)$.

(ii) If $a_{1} \ldots, a_{m+1}$ are arbitrary elements of $K^{\times}$, then $a_{1} \cup \ldots \cup a_{m+1}=0$ in $\mathrm{H}^{m+1}\left(G_{E}, \mathbb{Z} / \ell\right)$.

Proof. Under the hypothesis on the Lemma on has $\operatorname{vcd}_{\ell}(E)=\operatorname{cd}_{\ell}(E)$. By the discussion preceeding the Lemma, the only less obvious fact is to show that for $m=d+2$ the assertion (i) is satisfied. We show that this is true as follows: Let $Y$ be a model of $K$ over $\mathcal{O}_{\kappa}$. Let $x \in Y$ be a smooth point of $Y \rightarrow \mathcal{O}_{\kappa}$ with the properties:

(i) Its residue field $\kappa_{x}$ satisfies $\operatorname{char}\left(\kappa_{x}\right) \neq \ell$ and contains $\mu_{2 \ell}$.

(ii) $x$ is totally split in the field extension $E \mid K$.

(Such points $x$ do exist, why?) Let $\left(t_{d+1}, t_{d}, \ldots, t_{1}\right)$ be a regular sequence at $x$ such that $t_{d+1} \in \mathcal{O}_{\kappa}$. (This is possible, as $Y \rightarrow \mathcal{O}_{\kappa}$ is smooth at $x$.) The residue field $\kappa_{x}$ is a finite field. Choose $\bar{t}_{d+2} \in \kappa_{x}$ a non- $\ell^{\text {th }}$ power, and $t_{d+2} \in K$ a representative for it.

Claim: $t_{1} \cup \ldots \cup t_{d+2} \neq 0$ in $\mathrm{H}^{d+2}\left(G_{E}, \mathbb{Z} / \ell\right)$.

The idea of the proof is to construct an algebraic extension $\Lambda$ of $K$ containing $E$, and show that the restriction of $t_{1} \cup \ldots \cup t_{d+2}$ to $\Lambda$ is $\neq 0$, thus 
the same must hold over $E$. We take for $\Lambda$ a "multi-local field" at $x$. If namely $\mathfrak{p}$ is the place of $\kappa$ determined by the image of $x$ in $\operatorname{Spec} \mathcal{O}_{\kappa}$, and $\kappa_{\mathfrak{p}}$ is the completion of $\kappa$ at $\mathfrak{p}$, then $\Lambda \cong_{\kappa} \kappa_{\mathfrak{p}}\left(\left(t_{d}\right)\right) \ldots\left(\left(t_{1}\right)\right)$. Thus, if $v$ is the valuation of $\Lambda$, then

$$
v(K)=v(\Lambda)=: \Gamma \cong \mathbb{Z}^{d+1}
$$

with generators $v\left(t_{d+1}\right), v\left(t_{d}\right), \ldots, v\left(t_{1}\right)$. We also remark that the relative algebraic closure $K^{\mathrm{h}}$ of $K$ in $\Lambda$ is a Henselisation of $K$ at $v$. The absolute Galois group of $K^{\mathrm{h}}$ is canonically isomorphic to the absolute Galois group $Z=G_{\Lambda}$ of $\Lambda$. By the hypothesis on $x$, it follows that $E$ is $K$-embedable in $K^{\mathrm{h}} \subset \Lambda$. Further, by general valuation theory, see e.g. the explanations in [P], Local Theory, since $\mu_{2 \ell} \subset \Lambda$, we have:

1) Let $Z(\ell)$ and $G(\ell)$ be the maximal pro- $\ell$ quotients of $Z$, respectively of $G_{\kappa_{x}} \cong \widehat{\mathbb{Z}}$. Then $Z(\ell)$ has a well known structure given as follows: If $T(\ell)$ denotes the inertia subgroup of $Z(\ell)$, then one has a split group extension

$$
1 \rightarrow T(\ell) \rightarrow Z(\ell) \rightarrow G(\ell) \rightarrow 1
$$

Further, $T(\ell)$ is canonically ismorphic as a $G(\ell)$-module to $\operatorname{Hom}\left(\widetilde{\Gamma}, \mu_{\infty}\right)$, where $\widetilde{\Gamma}=(\Gamma \otimes \mathbb{Q}) / \Gamma$, and $\mu_{\infty}$ is the group of roots of unity whose orders are $\ell$-powers. Thus $Z(\ell)$ is isomorphic as an abstract profinite group to $\mathbb{Z}_{\ell}^{d+1} \rtimes G(\ell)$, where $G(\ell) \cong \mathbb{Z}_{\ell}$ acts on $\mathbb{Z}_{\ell}^{d+1}$ componentwise via the cyclotomic character of $G(\ell)$.

2 ) Let $Z_{\ell}$ denote a Sylow $\ell$-group of $Z$. Then the canonical projection $Z_{\ell} \rightarrow Z(\ell)$ is an isomorphism. See loc.cit..

In particular, the isomorphy type of $Z(\ell)$ depends only on $\operatorname{vcd}_{\ell}(K)$, and on the (cyclotomic character of the) residue field $\kappa_{x}$ of $x$. For the given residue field $\kappa_{x}$, thus the cyclotomic character of $G(\ell)$, and a given finite free $\mathbb{Z}_{\ell}$-module $\Delta_{n}$ of rang $(n-1)$ on which $G(\ell)$ acts via the cyclotomic character, we set $Z_{n}=\Delta_{n} \rtimes G(\ell)$. Thus the above $Z(\ell)$ is isomorphic as a profinite group to $Z_{d+2}$. The proof of the Claim will then follow from the following group theoretical fact:

Fact. One has $\operatorname{cd}_{\ell}\left(Z_{n}\right)=n$. More precisely, if $0 \neq \chi_{n} \in \mathrm{H}^{1}(G(\ell), \mathbb{Z} / \ell)$, and further $\chi_{1}, \ldots, \chi_{n-1} \in \mathrm{H}^{1}\left(\Delta_{n}, \mathbb{Z} / \ell\right)$ are independent characters, then $\chi_{1} \cup \ldots \cup \chi_{n} \neq 0$ and generates $\mathrm{H}^{n}\left(Z_{n}, \mathbb{Z} / \ell\right)$.

This is a well known fact, thus only sketch here a proof: Consider an exact sequence of the form $0 \rightarrow \Delta \rightarrow \Delta_{n} \rightarrow \Delta_{n-1} \rightarrow 0$ such that 
$\Delta \subset \operatorname{ker}\left(\chi_{i}\right)$ for $i<n-1$, and in particular, $\chi_{n-1}$ non-zero on $\Delta$. The above exact sequence gives rise to an exact sequence $0 \rightarrow \Delta \rightarrow Z_{n} \rightarrow Z_{n-1} \rightarrow 1$. Thus using for example a Hochschild-Serre spectral sequences of the form $\mathrm{H}^{p}\left(Z_{n-1}, \mathrm{H}^{q}(\Delta, \mathbb{Z} / \ell)\right) \Rightarrow H^{p+q}\left(Z_{n}, \mathbb{Z} / \ell\right)$, with a trivial action of $\Delta$ on $\mathbb{Z} / \ell$, one immediately obtains by induction the following: $\mathrm{H}^{n}\left(Z_{n}, \mathbb{Z} / \ell\right)$ is nontrivial, and it is generated by the cup-product $\chi_{1} \cup \chi_{2} \cup \ldots \cup \chi_{n}$.

To finish the proof of the above claim, we remark that in our context we can coose $\chi_{i}=\delta\left(t_{i}\right), i=1, \ldots, d+2$, where $\delta$ is the Kummer isomorphism. The hypotheses on the $\chi_{i}$ are satisfied, as by thier choice the elements $t_{1}, \ldots, t_{d+2}$ are independent in $\Lambda^{\times} / \ell$, and moreover, build an $\mathbb{F}_{\ell}$-basis of $\Lambda^{\times} / \ell$. Finally, point 2$)$ above implies that $\mathrm{H}^{i}(Z, \mathbb{Z} / \ell)$ and $\mathrm{H}^{i}(Z(\ell), \mathbb{Z} / \ell)$ are isomorphic.

\section{b) Transcendence degree and arithmetic}

As mentioned above, for every field $E$ there exists a canonical homomorphism called the tame residue symbol:

$$
h_{\ell, n}: \mathrm{K}_{n}^{\mathrm{M}}(E) / \ell \rightarrow \mathrm{H}^{n}\left(G_{K}, \mu_{\ell}^{\otimes n}\right)
$$

It is conjectured that $h_{\ell, n}$ is an isomorphism for all $\ell$ prime to $\operatorname{char}(E)$. This is a generalisation of the so called Milnor Conjecture, which is the above assertion for $\ell=2$. The point is that the Milnor Conjecture has a deep arithmetic significance relatetd to the arithmetic of the quadratic forms (for general $\ell$ we do not have yet an interpretation of the "generalised Minor Conjecture"). The Milnor conjecture is now proved by contributions of several people, with last major steps being done by Voevodsky, Rost, ET AL, see KAHN [Kh]. We describe below the facts which are significant for us.

With $E$ as above, let $W(E)$ be the Witt ring of $E$, i.e., the set of the isomorphy classes of anisotropic quadratic froms over $E$ with the usual addition and multiplication. Let $I(E)$ be the ideal of even dimensional quadradic forms, and $I^{n}(E)$ its powers. For $a_{1}, \ldots, a_{n}$ in $E^{\times}$let $<<a_{1}, \ldots, a_{n}>>$ be the corresponding ( $n$-fold) Pfister form. The set of all $n$-fold Pfister forms generates $I^{n}(E) / I^{n+1}(E)$. Milnor defined for every $n$ a homomorphism $d_{n}: \mathrm{K}_{n}^{\mathrm{M}}(E) / 2 \rightarrow I^{n}(E) / I^{n+1}(E)$ and conjectured that both $d_{n}$ and $h_{n}:=h_{2, n}$ are isomorphisms. In particular, this would give rise to higher cohomological invariants for quadratic forms, as we would then have then isomorphism $e_{n}: I^{n}(E) / I^{n+1}(E) \rightarrow \mathrm{H}^{n}\left(G_{K}, \mathbb{Z} / 2\right)$ for every $n$, thus generalising the $\operatorname{dim}(\bmod 2)$, the discriminant, and the Clifford (thus Hasse-Witt) 
invariant. The above isomorphism would work at the level of the Pfister forms as follows:

$$
e_{n}: \quad<<a_{1}, \ldots, a_{n}>>\mapsto\left(-a_{1}\right) \cup \ldots \cup\left(-a_{n}\right) .
$$

(Note that the minus-sign comes from a convention which is not necessarily the same in all sources. It depends on the definition of $\langle<a\rangle>$ which is $<\langle a\rangle\rangle=X_{0}^{2} \pm a X_{1}^{2}$. We work with the " + " convention.) See Elman-LAM [E-L], JAcobs-Rost, [J-R], etc. for more details and literature.

We recall the following fact: Let $q=<<a_{1}, \ldots, a_{n}>>$ be a given Pfister form, and $a \in E^{\times}$. Then $q$ represents $-a$ if and only if $q \otimes<<a>>$ is hyperbolic. Thus passing to Galois cohomology and using the Milnor Conjecture we get the following:

Fact. For a Pfister form $q=<<a_{1}, \ldots, a_{n}>>$ and $a \in E^{\times}$the following are equivalent:

(1) $q$ represents $-a$.

(2) $q \otimes\langle<a\rangle\rangle=\left\langle<a_{1}, \ldots, a_{n}, a\right\rangle>$ is hyperbolic.

(3) $\left(-a_{1}\right) \cup \ldots \cup\left(-a_{n}\right) \cup(-a)=0$ in $\mathrm{H}^{n+1}\left(G_{K}, \mathbb{Z} / 2\right)$.

Coming back to the proof of Fact 1.3, we remark that assertion (1) immediately follows from the discussion above with $E=K[\sqrt{-1}]$. To prove assertion (2), one proceeds by contradiction: Suppose that $\mathcal{B}_{d}=\left(t_{1}, \ldots, t_{d}\right)$ is not a transcendence basis of $K \mid \kappa$, and let $K_{0}=\kappa(\mathcal{B})$ be the subfield of $K$ generated by $\mathcal{B}$ over $\kappa$. Then $t\left(K_{0} \mid \kappa\right)<d$. Thus for all $t_{d+1}, t_{d+2} \in \kappa$ on has: The Pfister form $q_{\left(t_{1}, \ldots, t_{d+1}\right)}$ is universal over $K_{0}[\sqrt{-1}]$, in particular, it represents $t_{d+2}$. Therefore, $q_{\left(t_{1}, \ldots, t_{d+2}\right)}$ is hyperbolic over $K_{0}[\sqrt{-1}]$, thus over $K[\sqrt{-1}]$, contradiction !

Summarizing, we have the following way to describe the transcendence degree and even more, transcendence bases of a function field.

Theorem 1.4. Let $K \mid \kappa$ be a regular function field over some base field $\kappa$, where $\kappa$ is either algebraically closed, or finite, or a number field. To a system $\mathcal{B}=\left(t_{1}, \ldots, t_{r}\right)$ of elements of $K$ and a rational prime number $p$ let

$$
q_{\mathcal{B}_{r}}^{(p)}=\sum_{\underline{i}} \underline{t} \underline{i} X_{\underline{i}}^{p}
$$


be the homogenous form over $K$ whose coefficients are all the monomials of the form $\underline{t^{i}}=t_{1}^{i_{1}} \ldots t_{r}^{i_{r}}$ with $0 \leq i_{1}, \ldots, i_{r}<p$. Thus $q_{\mathcal{B}_{r}}:=q_{\mathcal{B}_{r}}^{(2)}$ is the $r$-fold Pfister form defined by $\mathcal{B}$. Then one has:

(1) Suppose $\kappa$ has characteristic $\operatorname{char}(\kappa)=p$. Then $\mathcal{B}_{d}$ is a separable transcendence basis of $K \mid \kappa$ if and only if $q_{\mathcal{B}_{d}}^{(p)}$ is universal, but does not represent 0 over $K$.

(2) Suppose $\kappa$ is algebraically closed with $\operatorname{char}(\kappa) \neq 2$. Then $\operatorname{td}(K \mid \kappa)=d$ if and only if $q_{\mathcal{B}_{d}}$ is universal over $K$ for every $\mathcal{B}_{d}$, and there does exist $\mathcal{B}_{d}$ such that $q_{\mathcal{B}_{d}}$ does not represent 0 over $K$.

(3) Suppose $\kappa$ is number field. Then $\operatorname{td}(K \mid \kappa)=d$ if and only if $q_{\mathcal{B}_{d+2}}$ is universal over $K[\sqrt{-1}]$ for every $\mathcal{B}_{d+2}$, and there does exist $\mathcal{B}_{d+2}$ such that $q_{\mathcal{B}_{d+2}}$ does not represent 0 over $K[\sqrt{-1}]$.

Moreover, $\left(t_{1}, \ldots, t_{d}\right)$ is a transcendence basis of $K \mid \kappa$ if there exit $t_{d+1}, t_{d+2} \in \kappa$ such that $q_{\left(t_{1}, \ldots, t_{d+2}\right)}$ does not represent 0 over $K[\sqrt{-1}]$. In particular, if $L \mid \lambda$ is another function field with $\lambda$ as above, such that $K$ and $L$ are elementarily equivalent, then one has: $\kappa$ and $\lambda$ are elementarily equivalent, thus they have the same characteristic; and they are isomorphic if $\kappa$ is finite or a number field; further $\operatorname{td}(K \mid \kappa)=\operatorname{td}(L \mid \lambda)$.

Proof. The assertions (1), (2), (3), are nothing but Facts 1.1, 1.2, 1.3 above. Concerning the last assertions, if $K$ and $L$ are elementarily equivalent, then $\kappa^{\text {abs }}=K^{\text {abs }} \cong L^{\text {abs }}=\lambda^{\text {abs }}$.

\section{Generalities about function fields}

In this section we put together well known facts about function fields and their models, facts which will be used later.

\section{A) Rings of definition}

Let $K \mid \kappa$ be an arbitrary, separably generated function field with $\kappa$ the constant field of $K$. Further let $X=\operatorname{Proj}(\kappa[\underline{X}] /(\underline{f}))$ be some projective model of $K \mid \kappa$. Here $\underline{X}=\left(X_{0}, \ldots, X_{n}\right)$ is a system of $(n+1)$ indeterminates, and $\underline{f}=\left(f_{1}, \ldots, f_{m}\right)$ is a system of homogenous polynomials in $\underline{X}$. We denote $\underline{x}=\left(x_{0}, \ldots, x_{n}\right)$ the resulting homogeneous coordinates on $X$.

In the above context, let $k$ be the prime field of $\kappa$. We define a ring of definition of $X \rightarrow \kappa$, and thus of $K \mid \kappa$, to be a $k$-subalgebra of finite type 
$R \subset \kappa$ over which the variety $X \rightarrow \kappa$ "is defined". The first approximation in doing this, is to take a $k$-subalgebra of finite type $R$ of $\kappa$ over which the ideal $(\underline{f})$ is defined, and set $\mathcal{X}=\operatorname{Proj}(R[\underline{X}] /(\underline{f}))$. By replacing $R$ by a bigger $k$-subalgebra of finite type of $\kappa$, we can suppose that $\mathcal{X} \rightarrow R$ and its fibers $X_{s}=\mathcal{X} \times_{R} \kappa_{s} \rightarrow \kappa_{s}$ at points $s \in \operatorname{Spec}(R)$ have as nice properties as the generic fiber $X \rightarrow \kappa$ has. Thus we can/will suppose that:

0) $\mathcal{X} \rightarrow R$ is flat.

1) Further, if $\kappa_{s}$ is the residue field at a point $s$ in $\operatorname{Spec}(R)$, then the fiber $X_{s}=\operatorname{Proj}\left(\kappa_{s}[\underline{X}] /\left(\underline{f}_{s}\right)\right) \rightarrow \kappa_{s}$ is geometrically irreducible.

Let $\mathcal{B}=\underline{t}=\left(t_{1}, \ldots, t_{d}\right)$ be a fixed (separable) transcendence basis of $K \mid \kappa$, and set $t_{\nu}=g_{\nu} / h_{\nu}$ with $g_{\nu}$ and $h_{\nu}$ homogeneous elements of $\kappa[\underline{X}] /(\underline{f})$ of the same degree. Then, after enlarging $R$, we can suppose that all the $g_{\nu}$ and $h_{\nu}$ are defined over $R$. We will say in this case that $\mathcal{B}$ is defined over $R$. Further, if $g_{\nu, s}$ and $h_{\nu, s}$ are the specializations of $g_{\nu}$ respectively $h_{\nu}$ at some $s \in \operatorname{Spec}(R)$, and the resulting rational functions $t_{\nu, s}=g_{\nu, s} / h_{\nu, s}$ on $X_{s}$ are defined, then we can consider the system $\mathcal{B}_{s}=\underline{t}_{s}=\left(t_{1, s}, \ldots, t_{d, s}\right)$ of rational functions in in the function field $K_{s}=\kappa\left(X_{s}\right)$ over $\kappa_{s}$. We will say that in this case that $\mathcal{B}_{s}$ is defined at $s$. In this context, after shrinking $\operatorname{Spec} R$, we can suppose that for all $s \in \operatorname{Spec}(R)$ it holds:

2) If $t_{\nu, s}=g_{\nu, s} / h_{\nu, s}$ are defined, and the system $\mathcal{B}_{s}=\underline{t}_{s}=\left(t_{1, s}, \ldots, t_{d, s}\right)$ is a (separable) transcendence basis of the function field $K_{s}=\kappa_{s}\left(X_{s}\right)$ over $\kappa_{s}$.

In particular, we can/will suppose that the above condition is satisfied at every $s \in \operatorname{Spec}(R)$, and that we have $[K: \kappa(\mathcal{B})]=\left[K_{s}: \kappa_{s}\left(\mathcal{B}_{s}\right)\right]$.

3) If $X$ is (projectively) normal, then the same is true for $\mathcal{X}$, and for its fibers $X_{s}$.

4) If $X \rightarrow \kappa$ is smooth, the same is true for $\mathcal{X} \rightarrow R$. In particular, the fibers $X_{s} \rightarrow \kappa_{s}$ are then projective and smooth.

Now suppose that $X \rightarrow \kappa$ is smooth, and by the remarks above, the same is true for $\mathcal{X} \rightarrow R$. This means that the sheaf of the relative differentials $\Omega_{\mathcal{X} \mid R}$ is locally free of rank $d=\operatorname{td}(K \mid \kappa)$, thus its $d^{\text {th }}$ exterior power $\omega_{\mathcal{X} \mid R}$ is an invertible sheaf on $\mathcal{X}$ (the relative canonical class). Taking this into account and using the Semi-continuity Theorem, after again shrinking $\operatorname{Spec} R$, we get: 
5) Given a finite set $\mathfrak{I}$ of birational invariants, like for instance plurigenera and/or Hodge numbers of $X$, it follows that the special fibers $X_{s}$ have the same birational invariants.

6) In particular, if $\mathcal{D}$ is a (relative) effective divisor in the divisor class of $\omega_{\mathcal{X} \mid R}$, and $D$ and $D_{s}$ are its generic fiber respectively its fiber at $s$, then the linear spaces $|m \mathcal{D}|,|m D|$, and $\left|m D_{s}\right|$ have the same dimension (for $m$ in some range $0 \leq m \leq n$ ), and the canonical $R$-morphism $\mathcal{X} \rightarrow \mathbb{P}_{R}^{N}$ with $N$ the dimension of $|m \mathcal{D}|$, give rise by base change to the corresponding canonical projective $\kappa$-morphism $X \rightarrow \mathbb{P}_{\kappa}^{N}$ respectively $\kappa_{s}$-morphism $X_{s} \rightarrow \mathbb{P}_{\kappa_{s}}^{N}$.

B) Approximations of function fields

Let $K \mid \kappa$ and $\Lambda \mid \lambda$ be function fields.

Let $\mathcal{X} \rightarrow R$ be a model of $K \mid \kappa$ as above. We define an approximation of $K \mid \kappa$ with values in $\Lambda \mid \lambda$ via $\mathcal{X} \rightarrow R$ to be a $\Lambda$-rational point $\varphi: \operatorname{Spec} \Lambda \rightarrow \mathcal{X}$ of $\mathcal{X}$ with the property: There exists a morphism of rings $p: R \rightarrow \lambda$ such that denoting $X_{\varphi}=\mathcal{X} \times_{R} \lambda$ the base change of $\mathcal{X}$ to $\lambda$ via $p$, the point $\varphi$ is defined by a $\lambda$-embedding of function fields $\imath_{\varphi}: \lambda\left(X_{\lambda}\right) \rightarrow \Lambda$. This implies in particular that $\operatorname{td}(K \mid \kappa)=\operatorname{td}\left(\lambda\left(X_{\varphi}\right) \mid \lambda\right) \leq \operatorname{td}(\Lambda \mid \lambda)$. For an approximation $\varphi$ of $K \mid \kappa$ with values in $\Lambda \mid \lambda$ via some given model $\mathcal{X} \rightarrow R$ as above we now make the following definitions:

- Let $\mathcal{B}_{d}=\left(t_{1}, \ldots, t_{d}\right)$ be a separable transcendence base of $K \mid \kappa$ as in subsection A), 2) above. We say that $\varphi$ is $\mathcal{B}$-separable, if $\Lambda \mid \lambda$ is separably generated, and $\iota_{\varphi}(\mathcal{B})$ is contained in a separable transcendence base of $\Lambda \mid \lambda$.

- We will say that $\varphi$ is smooth, if $\mathcal{X} \rightarrow R$ is a smooth morphism. In particular, $X_{\varphi} \rightarrow \lambda$ is a smooth morphism.

- We will say that $\varphi$ is minimal, if $\operatorname{dim}(R)$ is minimal among the dimensions of all the possible rings of definition of $K \mid \kappa$.

- We will say that $K \mid \kappa$ has enough (separable) approximations with values in $\Lambda \mid \lambda$, if for every model $\mathcal{X} \rightarrow R$ of $K \mid \kappa$ (endowed with a separable transcendence basis $\mathcal{B}$ ) as above, $K \mid \kappa$ has ( $\mathcal{B}$-separable) approximations with values in $\Lambda \mid \lambda$ via $\mathcal{X} \rightarrow R$.

Theorem 2.1. In the above notations the following holds:

(1) Let $K|\kappa, \Lambda| \lambda, \Lambda^{\prime} \mid \lambda^{\prime}$ be function fields. Suppose that $K \mid \kappa$ has enough (separable) approximations with values in $\Lambda \mid \lambda$, and that $\Lambda \mid \lambda$ has enough 
(separable) approximations with values in $\Lambda^{\prime} \mid \lambda^{\prime}$. Then $K \mid \kappa$ has enough (separable) approximations with values in $\Lambda^{\prime} \mid \lambda^{\prime}$.

(2) Let $K \mid \kappa$ and $L \mid \lambda$ be function fields such that both: $K \mid \kappa$ has enough separable smooth approximations with values in $L \mid \lambda$, and $L \mid \lambda$ has enough separable approximations with values in $K \mid \kappa$. Further suppose that $K \mid \kappa$ is a function field of general type. Then $K \mid \kappa$ and $L \mid \lambda$ have isomorphic function subfields $\imath_{0}: K_{0}\left|\kappa_{0} \rightarrow L_{0}\right| \lambda_{0}$ such that $K=K_{0} \kappa$ and $L=L_{0} \lambda$.

In particular, if the isomorphism $\imath_{0}: \kappa_{0} \rightarrow \lambda_{0}$ can be prolonged to an isomorphism $\kappa \cong \lambda$, then $\imath_{0}$ has a prolongation to an isomorphism of function fields $\imath: K|\kappa \rightarrow L| \lambda$.

Proof. To (1): Let $R \subset \kappa, \mathcal{X} \rightarrow R$ (and a transcendence base $\mathcal{B}$ of $K \mid \kappa$ as in subsection A), 2) above) be given. We show that $K \mid \kappa$ has (Bseparable) approximations with values in $\Lambda^{\prime} \mid \lambda^{\prime}$ via $\mathcal{X} \rightarrow R$. In the above notations, let $\varphi: \Lambda \rightarrow \mathcal{X}$ be a ( $\mathcal{B}$-separable) approximation of $K \mid \kappa$ with values in $\Lambda \mid \lambda$. Let $p: R \rightarrow \lambda$ be a ring homomorphism defined by $\varphi$, and $\imath_{\varphi}: \lambda\left(X_{\varphi}\right) \rightarrow \Lambda \mid \lambda$ the $\lambda$-embedding of function fields defined by $\varphi$. By general facts concerning rational dominant maps we get: Given any model $Y \rightarrow \lambda$ of $\Lambda \mid \lambda$, the morphism of function fields $\imath_{\varphi}$ is defined by a rational map $\phi: Y-->X_{\varphi}$. Moreover, there exist projective models $Y \rightarrow \lambda$ of $\Lambda \mid \lambda$ such that $\imath_{\varphi}$ is induced by a dominant $\lambda$-morphism $\phi_{\varphi}: Y \rightarrow X_{\varphi}$. Let now $S \subset \lambda$ be a finitely generated $k$-subalgebra containing $p(R)$, over which $Y$ and the morphism $\phi_{\varphi}$ are defined. This means, there exits a model $\mathcal{Y} \rightarrow S$ of $\Lambda \mid \lambda$ whose base change to $\lambda$ is $Y \rightarrow \lambda$, togehter with a morphism $\phi: \mathcal{Y} \rightarrow \mathcal{X}$ which defines $\varphi$ as the composition

$$
\varphi: \operatorname{Spec} \Lambda \stackrel{\operatorname{can}}{\longrightarrow} \mathcal{Y} \stackrel{\phi}{\longrightarrow} \mathcal{X}
$$

We can/will suppose that $\mathcal{Y} \rightarrow S$ satisfies the conditions 0) and 1) from subsection A) above. Further, if the approximation $\varphi$ was $\mathcal{B}$-separable, let $\mathcal{C}$ be a separable transcendence basis of $\Lambda \mid \lambda$ containing $\imath_{\varphi}(\mathcal{B})$. Then we can/will suppose that condition 2) of subsection A) is satisfied for $\mathcal{Y} \rightarrow S$ endowed with $\mathcal{C}$.

Now let $\mathcal{X}_{S}=\mathcal{X} \times{ }_{R} S$ be the base change of $\mathcal{X} \rightarrow R$ to $S$ via $p: R \rightarrow S$. Then there exists a dominant $S$-morphism $\phi_{S}: \mathcal{Y} \rightarrow \mathcal{X}_{S}$, whose base change 
to $\lambda$ is the given $\lambda$-morphism $\phi_{\varphi}: Y \rightarrow X_{\varphi}$. Thus in particular, $\phi_{S}$ defines the function field homomorphism $\imath_{\varphi}$.

In order to finish the proof, let $\varpi: \operatorname{Spec} \Lambda^{\prime} \rightarrow \mathcal{Y}$ be a $(\mathcal{C}$-separable $)$ approximation of $\Lambda \mid \lambda$ with values in $\Lambda^{\prime} \mid \lambda^{\prime}$ via $\mathcal{Y} \rightarrow S$. On then checks without difficulty that the composition

$$
\varphi^{\prime}: \operatorname{Spec} \Lambda^{\prime} \stackrel{\varpi}{\longrightarrow} \mathcal{Y} \stackrel{\phi_{S}}{\longrightarrow} \mathcal{X}_{S} \stackrel{\text { can }}{\longrightarrow} \mathcal{X}
$$

is a ( $\mathcal{B}$-separable) approximation of $K \mid \kappa$ with values in $\Lambda^{\prime} \mid \lambda^{\prime}$ via $\mathcal{X} \rightarrow R$.

To (2). We first remark that the existence of approximations of both: $K \mid \kappa$ with values in $L \mid \lambda$, and vice-versa, implies $\operatorname{td}(K \mid \kappa)=\operatorname{td}(L \mid \lambda)$.

In the notations from the proof of (1) above, we replace $\Lambda \mid \lambda$ by $L \mid \lambda$, and $\Lambda^{\prime} \mid \lambda^{\prime}$ by $K \mid \kappa$. For all possible separable transcendence bases $\mathcal{B}$ of $K \mid \kappa$, we consider all $\mathcal{B}$-separable approximations of $K \mid \kappa$ with values in $L \mid \kappa$ via such $\mathcal{X} \rightarrow R$ that $\mathcal{X} \rightarrow R$ satisfies the properties 0)-6) of subsection A). Among all these possible choices we consider the ones in which $\operatorname{dim}(R)$ is minimal.

In the notations from the proof of (1) above, and the hypothesis of (2), if $\varphi$ is a $\mathcal{B}$-separable approximation, then $\mathcal{C}=\varphi(\mathcal{B})$ is a separable transcendence basis of $L \mid \lambda$. Now let $\varpi: K \rightarrow \mathcal{Y}$ be a $\mathcal{C}$-separable approximation of $L \mid \lambda$ with values in $K \mid \kappa$ via $\mathcal{Y} \rightarrow S$. (Such approximations do exist, as $L \mid \lambda$ is supposed to have enough separable approximations with values in $K \mid \kappa$.) As in the proof of assertion (1), there exists a projective model $\mathcal{X}^{\prime} \rightarrow R^{\prime}$ of $K \mid \kappa$, and a $k$-homomorphism $q: S \rightarrow R^{\prime}$ together with an homomorphism of $R^{\prime}$-varieties $\psi_{R^{\prime}}: \mathcal{X}^{\prime} \rightarrow \mathcal{Y}_{R^{\prime}}$, such that $\varpi$ is the base change of $\psi_{R^{\prime}}$ to $\kappa$ via the inclusion $R^{\prime} \hookrightarrow \kappa$. Putting everything together, we obtain a smooth, $\mathcal{B}$-separable approximation of $K \mid \kappa$ with values in $K \mid \kappa$ which is defined via the composition $p^{\prime}: R \stackrel{p}{\longrightarrow} S \stackrel{q}{\longrightarrow} R^{\prime}$ as follows

$$
\varphi^{\prime}: \operatorname{Spec} K \hookrightarrow \mathcal{X}^{\prime} \stackrel{\phi_{R^{\prime}}}{\longrightarrow} \mathcal{Y}_{R^{\prime}} \stackrel{\text { can }}{\longrightarrow} \mathcal{Y} \stackrel{\phi_{S}}{\longrightarrow} \mathcal{X}_{S} \stackrel{\text { can }}{\longrightarrow} \mathcal{X}
$$

In the above context, let $\kappa_{0}=\operatorname{Quot}(R)$, and $K_{0} \mid \kappa_{0}$ be the function field of $\mathcal{X} \rightarrow R$. Thus $K=K_{0} \kappa$ is the compositum of $K_{0}$ and $\kappa$ over $\kappa_{0}$ inside $K$. We now have:

Claim 1. $p^{\prime}$ is injective, and the $\kappa$-homomorphism $\imath_{\varphi^{\prime}}: \kappa\left(X_{\varpi^{\prime}}\right) \rightarrow K$ is an isomorphism. Equivalently, $K=\imath_{\varphi^{\prime}}\left(K_{0}\right) \kappa$ inside $K$.

Proof of Claim 1. Let $X^{\prime}=\mathcal{X}^{\prime} \times_{R^{\prime}} \kappa$, and $\phi_{\varphi^{\prime}}: X^{\prime} \rightarrow X_{\varphi^{\prime}}$ the base change to $\kappa$ of the $R^{\prime}$-morphism $\mathcal{X}^{\prime} \rightarrow \mathcal{X}_{R^{\prime}}$ from above. Since both $\varphi$ and 
$\varpi$ were -among other things- separable approximations, it follows that $\phi_{\varphi^{\prime}}$ is a generically finite and separable $\kappa$-morphism; and it defines the above homomorphism of function fields $\imath_{\varphi^{\prime}}: \kappa\left(X_{\varpi^{\prime}}\right) \rightarrow K$. Let $X \rightarrow \kappa$ be the generic fiber of $\mathcal{X} \rightarrow R$. Then $X$ is birationally equivalent to $X^{\prime}$. Therefore, $\imath_{\varphi^{\prime}}$ is defined by some dominant generically finite and separable rational $\kappa$-map $\phi^{\prime}: X-->X_{\varpi^{\prime}}$. Thus we have the following situation:

- Both $X \rightarrow \kappa$ and $X_{\varpi^{\prime}} \rightarrow \kappa$ are projective smooth varieties of general type, and $\phi^{\prime}: X-->X_{\varpi^{\prime}}$ is a generically finite and separable rational $\kappa$-map.

- Let $\omega_{X}$ and $\omega_{X_{\varpi^{\prime}}}$ be their canonical classes. Then for all $m>0$ some given range $m \leq n$, we have $\operatorname{dim}\left|\omega_{X}^{\otimes m}\right|=\operatorname{dim}\left|\omega_{X_{\varpi^{\prime}}}^{\otimes m}\right|=: N_{m}$.

We now choose from the beginning $n$ sufficiently large, such as to obatain an $n$-canonical embeddings in $\mathbb{P}_{\kappa}^{N}$, where $N=N_{n}$. Thus the same is true for a model $\mathcal{X} \rightarrow R$ of $X \rightarrow \kappa$ which satisfies the conditions 0 )-6) of subsection A) above. In particular, the same is true for $X_{\varphi^{\prime}}$, as it is a base change of fibers of $\mathcal{X} \rightarrow R$.

Now using e.g. Iitaka [I], Ch.5, $\S 5.4$, it follows that in this situation, every dominant rational separable $\kappa$-map $\phi^{\prime}: X-->X_{\varpi^{\prime}}$ is birational.

In order to finish the proof of the Claim, we remark that denoting $\bar{R}=p^{\prime}(R)$ the image of $R$ under $p^{\prime}$ (which is the same as the image of $R$ under $\imath_{\varphi^{\prime}}$ ), it follows that $\overline{\mathcal{X}}=\mathcal{X} \times{ }_{R} \bar{R}$ has the property: $\overline{\mathcal{X}} \times \bar{R} \kappa=X_{\varphi^{\prime}}$. Since $\kappa\left(X_{\varphi^{\prime}}\right)=K$, it follows that $\overline{\mathcal{X}} \rightarrow \bar{R}$ is a projective smooth model of $K \mid \kappa$. Further, $\overline{\mathcal{B}}=\imath_{\varphi^{\prime}}(\mathcal{B})$ is defined over $\bar{R}$, and $\overline{\mathcal{X}}=\mathcal{X} \times{ }_{R} \bar{R}$ endowed with $\overline{\mathcal{B}}$ has the properties 0 )-6) from subsection A) above. By the minimality of $\operatorname{dim}(R)$, it follows that $\operatorname{dim}(R)=\operatorname{dim}(\bar{R})$, thus $p^{\prime}$ maps $R$ isomorphically onto $\bar{R}=p^{\prime}(R)$.

Now let $\mathcal{X} \rightarrow R$ a projective smooth model of $K \mid \kappa$ endowed with a separable transcendence basis $\mathcal{B}$, such that $\operatorname{dim}(R)$ is minimal, and the conditions 0)-6) of subsection A above are satisfied.

Claim 2. Let $\varphi: L \rightarrow \mathcal{X}$ be a $\mathcal{B}$-separable approximation of $K \mid \kappa$ via $\mathcal{X} \rightarrow R$. Then $\varphi$ is dominant, and the corresponding $\imath_{\varphi}: \lambda\left(X_{\varphi}\right) \rightarrow L$ is an isomorphism. In particular, $L \mid \lambda$ is a function field of general type, and $X_{\varphi}$ is a projective smooth model of $L \mid \lambda$.

Proof of Claim 2. Let us denote $\mathcal{C}=\imath_{\varphi}(\mathcal{B})$. Then $\mathcal{C}$ is a (separable) transcendece basis of $L \mid \lambda$. Moreover, since $\mathcal{X} \rightarrow R$ has property 2$)$ of subsection A), it follows that $[K: \kappa(\mathcal{B})]=\left[\lambda\left(X_{\varphi}\right): \lambda(\mathcal{C})\right]$. Thus $\imath_{\varphi}: \lambda\left(X_{\varphi}\right) \rightarrow L$ 
is an isomorphism $\Leftrightarrow[K: \kappa(\mathcal{B})]=[L: \lambda(\mathcal{C})]$. By Claim 1 (and in the notations from their), it follows that $p^{\prime}: R \rightarrow S \rightarrow R^{\prime}$ is injective, thus $p: R \rightarrow S$ is injective. Equivalently, $\varphi: L \rightarrow \mathcal{X}$ factoriezes through the generic point of $R$. In other words, denoting $\kappa_{0}=\operatorname{Quot}(R)$, and by $K_{0}$ the function field of $\mathcal{X}$ we have:

- $K=K_{0} \kappa$ in a canonical way.

- $\imath_{\varphi}: K_{0}\left|\kappa_{0} \rightarrow L\right| \lambda$ is a morphism of function fields.

- $\mathcal{C}=\imath_{\varphi}(\mathcal{B})$ is a separable transcendence basis of $L \mid \lambda$.

Now in the notations from the proof of assertion (1) above, suppose that the model $\mathcal{Y} \rightarrow S$ endowed with the separable transcendence basis $\mathcal{C}$ of $L \mid \lambda$ satisfies condition 2) of subsection A). Then $\mathcal{B}^{\prime}:=\imath_{\varpi}(\mathcal{C})$ is a separable transcendence basis of $K \mid \kappa$ such that $\left[\kappa\left(X_{\varpi}\right): \kappa\left(\mathcal{B}^{\prime}\right)\right]=[L: \lambda(\mathcal{C})]$.

Let us set $L_{0} \mid \lambda_{0}=\imath_{\varphi}\left(K_{0} \mid \kappa_{0}\right)$. By Claim 1 , on has $K=\kappa\left(X_{\varpi}\right)$, and therefore also $\left[K: \kappa\left(\mathcal{B}^{\prime}\right)\right]=[K: \kappa(\mathcal{B})]$. Hence we have $[K: \kappa(\mathcal{B})]=[L: \lambda(\mathcal{C})]$, or equivalently, $L=L_{0} \lambda$.

The proof of assertion (2) is completed. Thus Theorem 2.1 is proved.

\section{Proof of Theorem A and Theorem B}

Both in the context of Theorem A and Theorem B, let $\Psi:{ }^{*} K \rightarrow{ }^{*} L$ be a fixed isomorphism of some ultra-powers ${ }^{*} K=K^{I} / \mathcal{D}$ and ${ }^{*} L=L^{J} / \mathcal{E}$ of $K$ respectively $L$. We set ${ }^{*} \kappa$ and ${ }^{*} \lambda$ for the corresponding ultra-powers of $\kappa$ and $\lambda$ inside ${ }^{*} K$, respectively ${ }^{*} L$. We think about $K \mid \kappa$ and $L \mid \lambda$ as being diagonally emebdded in ${ }^{*} K$, respectively ${ }^{*} L$. The following holds:

Fact 3.1. In the above kontext one has:

(1) If $\kappa$ is a number field or a finite field, then $\Psi(\kappa)=\lambda$.

(2) If $\kappa$ is algebraiclly closed, then the same is true for $\lambda$, and $\Psi\left({ }^{*} \kappa\right)={ }^{*} \lambda$.

Proof. The first assertion is clear. The second might be also known, but we cannot give a reference. It follows immediately from Lemma 3.3 below (which itself relies on the next Lemma 3.2).

We begin by a little preparation. Let $\mathbb{P}_{t}^{1}$ be the $t$-projective line over $\kappa$, and $K_{0}=\kappa(t)$ its function field. For every $a \in \kappa$ we denote by $K_{a} \mid K_{0}$ a minimal field extension of $K_{0}$ in which $p_{a, t}(T) \in K_{0}[T]$ has a root, where

a) $p_{a, t}(T)=T^{2}-1 /(t-a)$, if $\operatorname{char}(\kappa) \neq 2$ 
b) $p_{a, t}(T)=T^{2}-T-1 /(t-a)$, if $\operatorname{char}(\kappa)=2$

Let $C_{a} \rightarrow \mathbb{P}_{t}^{1}$ be the normalisation of $\mathbb{P}_{t}^{1}$ in the Galois field extension $K_{a} \mid K_{0}$. It is clear that $C_{a} \rightarrow \mathbb{P}_{t}^{1}$ is ramified exactly in $t=a, \infty$ in case a), respectively in $t=a$ in case b). Thus, if $S \subset \kappa$ is a finite subset of cardinality $n>0$, then the field extensions $K_{a} \mid K_{0}$ are linearly disjoint over $K_{0}$, and their compositum $K_{S} \mid K_{0}$ is the function field of the fiber product $C_{S}$ of all the $C_{a}$ over $\mathbb{P}_{t}^{1}$. Using the Hurwitz genus formula, we se that the genus $g_{n}$ of $C_{S}$ is given by

a) $g_{n}=2^{n-2}(n-3)+1$, if $\operatorname{char}(\kappa) \neq 2$

b) $g_{n}=2^{n-1}(n-2)+1$, if $\operatorname{char}(\kappa)=2$,

thus it depends only on $n$ and on $\operatorname{char}(\kappa)$. The main technical point (which on the other hand might be well known to specialists, but again, we cannot give a reference) is the following:

Lemma 3.2. Let $K \mid \kappa$ be a function field with $\kappa$ algebraically closed. For $x \in K$ let $S_{x}$ be the set of all $a \in \kappa, a \neq x$, such that $p_{a, x}(T)$ has a root in $K$. Then there exits a bound $c=c_{K \mid \kappa}$ with the property: If $x \in K$ is a non-constant function, then the cardinality of $S_{x}$ is bounded by $c$. To the contrary, if $x \in \kappa$, then $S_{x}=\kappa \backslash\{a\}$, thus infinite.

Proof of Lemma 3.2. We make induction on $d=\operatorname{td}(K \mid \kappa)$. Let $X \rightarrow \kappa$ be a projective normal model of $K \mid \kappa$, and let $\imath: X \hookrightarrow \mathbb{P}_{\kappa}^{N}$ be a projective embedding of $X$. Then for every $d-1$ hyper-planes $H_{i}$ in "general position" in $\mathbb{P}_{\kappa}^{N}$, let $C=X \cap_{i} H_{i}$ be the resulting curve in $X$. It is well known that the following holds:

- $C \rightarrow \kappa$ is a normal curve.

- The set of generic points $\eta_{C}$ of all the generic curves $C$ is dense in $X$.

- The genus $g$ of $C$ is independent of the concrete choice of the hyperplanes, it being an invariant of the projective embedding $\imath$.

We will show that we can take $c=c_{K \mid \kappa}=g+2$.

For every non-constant function $x \in K$, let $K_{x}$ be the relative algebraic closure of $\kappa(x)$ in $K$. Further, let $C_{x} \rightarrow \kappa$ be a projective normal (thus smooth) model of $K_{x} \mid \kappa$. For a be a finite subset $S$ of $S_{x}$, let $K_{S} \subset K_{x}$ be the extension of $\kappa(x)$ generated by the roots of all the $p_{a, x}(T)$ with $a \in S$. Thus if $C_{S}$ is a projective normal model for $K_{S}$, then there exists a dominant $\kappa$-homomorphism $C_{x} \rightarrow C_{S}$. Furthermore, if $X \rightarrow \kappa$ is a normal model for 
$K \mid \kappa$, then the inclusion $K_{x} \rightarrow K$ is defined by a dominant rational $\kappa$-map $f: X-->C_{x}$.

First suppose $\operatorname{td}(K \mid \kappa)=1$. Then $C_{x}=X$ is a projective normal model for $K \mid \kappa$, and $g$ is the genus of $X$. Since $X=C_{x} \rightarrow C_{S}$ is dominant, by the Hurwitz genus formula, $g \geq g_{n}$, where $n=|S|$ is the cardinality of $S$. Since $g_{n}+2 \geq n$, we deduce that all finite subsets $S$ of $S_{x}$ have cardinality bounded by $g+2$.

Coming to the general case, since the set of the generic points $\eta_{C}$ is dense in $X$, it follows that there exit points $\eta_{C}$ at which $f: X-->C_{x}$ is defined. This means that $f$ defines a rational $\kappa$-map $f_{x}: C-->C_{x}$ of projective normal curves. Again, by the the Hurwitz genus formula, it follows that $g \geq g_{x}$ with $g_{x}$ the genus of $C_{x}$. We conclude as above.

The proof of the Lemma 3.2 is finished.

Lemma 3.3. Let $K \mid \kappa$ be a function with $\kappa$ an algebraically closed field. Let further ${ }^{*} K=K^{I} / \mathcal{D}$ be some ultra-power of $K$. Then ${ }^{*} \kappa$ is the unique maximal algebraically closed subfield of ${ }^{*} K$.

More precisely, the description of ${ }^{*} \kappa$ inside ${ }^{*} K$ can be given as follows: For every ${ }^{*} x \in{ }^{*} K$ let $S_{*_{x}} \subset \kappa^{\text {abs }}$ be the set of all absolute algebraic elements of $\kappa$ for which $p_{a, x}(T)$ has roots in ${ }^{*} K$. Then one has:

(i) $\left|S_{*}\right| \leq c_{K \mid \kappa}$ for all ${ }^{*} x \notin{ }^{*} \kappa$, in particular $\left|S_{*}\right|$ is finite.

(ii) $\left|S^{*}\right|=\kappa^{\text {abs }} \backslash\left\{{ }^{*} x\right\}$ for all ${ }^{*} x \in{ }^{*} \kappa$, in particular $\left|S^{*}\right|$ is infinite.

Proof of Lemma 3.3 immediately follows from Lemma 3.2, thus we will omit the proof here.

Finally, the proof of the second assertion of Fact 3.1 follows from Lemma 3.3 and the observation that $\Psi\left(\kappa^{\text {abs }}\right)=\lambda^{\text {abs }}$ inside $\Psi\left({ }^{*} K\right)={ }^{*} L$.

We now come to the Proof of Theorem $A$ and Theorem B.

We first remark that assertion (1) of both Theorem A and Theorem B follows from Theorem 1.4 using Fact 3.1 above. Indeed, suppose first that $\operatorname{char}(\kappa)=p$ is positive. Then $\operatorname{char}(\lambda)=p$ is positive. Further, Theorem 1.4, (1), asserts: $\operatorname{td}(K \mid \kappa)=d$ is equivalent to saying that $\exists \mathcal{B}_{d}=\left(t_{1}, \ldots, t_{d}\right)$ which is $p$-base of $K$. Moreover, if this assertion is true for $\mathcal{B}_{d}$, then $\mathcal{B}_{d}$ is a separable transcendence basis of $K \mid \kappa$. The case $\kappa$ is algebraically closed of 
characteristic zero is completely similar. Now suppose that $\kappa$ is a number field. Then $\lambda$ is a number field too, as $\kappa={ }^{*} \kappa^{\text {abs }}$, and $\lambda={ }^{*} \lambda^{\text {abs }}$, and $\Psi\left({ }^{*} \kappa^{\mathrm{abs}}\right)={ }^{*} \lambda^{\mathrm{abs}}$. Moreover, using Theorem 1.4, (3), it follows that $\operatorname{td}(K \mid \kappa)=\operatorname{td}(L \mid \lambda)$.

We now come to the proof of assertion (2) of Theorem A and Theorem B. We will be using Theorem 2.1. In order to do this, we will show that -roughly speaking- elementary equivalence implies existence of enough approximations, even without the supplementary condition on $K \mid \kappa$ of being of general type.

Key Lemma. Let $K \mid \kappa$ and $L \mid \lambda$ be function fields over either finite fields, or number fields, or algebraically closed fields. Suppose that $K$ and $L$ are elementarily equivalent. Then $K \mid \kappa$ has enough separable approximations with values in $L \mid \lambda$, and $L \mid \lambda$ has enough separable approximations with values in $K \mid \kappa$.

Proof. Let $\Psi:{ }^{*} K \rightarrow{ }^{*} L$ be an isomorphism of some ultra-powers of $K$ and $L$. We will show that $\Psi$ gives rise to enough approximations of $K \mid \kappa$ with values in $L \mid \lambda$.

Let $\mathcal{B}=\left(t_{1}, \ldots, t_{d}\right)$ be a separable transcendence basis of $K \mid \kappa$, and further $\mathcal{X}=\operatorname{Proj}(R[\underline{X}] /(\underline{f})) \rightarrow R$ some model of $K \mid \kappa$, where $R=k[\underline{y}]$ is a finitely generated $k$-algebra, $k$ being the prime field of $\kappa$ and $\lambda$. We will suppose that $\mathcal{X} \rightarrow R$ satisfies/has the properties 0 )-4) of Section 2, A), respectively properties 0)-6) if it is the case.

Without loss of generality, suppose that all the polynomials in the system $\underline{f}$ are absolutely irreducible, and $X_{0}$ is not among them. Then we have $K=\kappa(\underline{u})$, where $\underline{u}=\left(x_{1} / x_{0}, \ldots, x_{n} / x_{0}\right)$ is the "canonical system" of rational functions on $\mathcal{X}$ generating $K \mid \kappa$.

Via the the restriction $p=\left.\Psi\right|_{R}$, we get an embedding $p: R \rightarrow{ }^{*} \lambda$. Unfortunately we do not know a priori that $S:=p(R) \subset \lambda \ldots$ Via $p$ we can consider the base change ${ }^{*} \mathcal{X}=\mathcal{X} \times_{R} S \rightarrow S \subset{ }^{*} \lambda$. This is nothing but $* \mathcal{X}=\operatorname{Proj}(S[\underline{X}] / p(\underline{f})) \rightarrow S$.

Next, setting $R=k[\underline{y}]$, and taking local representatives $\underline{y}_{j} \in \lambda$ for $p(\underline{y})$, it follows that locally we get local representatives $p_{j}: R \rightarrow S_{j} \subset \lambda$ for the ring homomorphism $p: R \rightarrow S \subset{ }^{*} \lambda$. 
Considering "local representatives" $\underline{f}_{j}$ for $p(\underline{f})$, we get local representatives $\mathcal{X}_{j} \rightarrow S_{j}$ for ${ }^{*} \mathcal{X} \rightarrow S \subset{ }^{*} \lambda$, which are defined by

$$
\mathcal{X}_{j}=\operatorname{Proj}\left(S_{j}[\underline{X}] /\left(\underline{f}_{j}\right)\right) \rightarrow S_{j}
$$

We remark that $\mathcal{X}_{j} \rightarrow S_{j}$ is nothing but the base change $\mathcal{X} \times_{R} S_{j} \rightarrow S_{j}$ of $\mathcal{X} \rightarrow R$ to $S_{j}$ via the local morphisms $p_{j}: R \rightarrow S_{j}$. Further, by the usual properties of ultra-products we have: If $\underline{u}_{j}$ as systems of elements in $L$ are local representatives for $\Psi(\underline{u})$ as systems of elements of ${ }^{*} L$, then locally $\left(1, \underline{u}_{j}\right)$ is a zero of the the homogeneous system of equations $\underline{f}_{j}=0$ over $S_{j}$. Moreover, for $1 \leq \nu \leq d$ let $t_{\nu}=g_{\nu}(\underline{x}) / h_{\nu}(\underline{x})$ be fixed representations of $t_{\nu}$ with $g_{\nu}(\underline{X})$ and $h_{\nu}(\underline{X})$ homogenous equal degree polynomials over $R$. Then $g_{j \nu}=p_{j}\left(g_{\nu}\right)$ and $h_{j \nu}=p_{j}\left(h_{\nu}\right)$ are homogenous equal degree polynomials over $S_{j}$ which locally are representatives for $p\left(g_{\nu}\right)$, respectively $p\left(h_{\nu}\right)$, and further holds: $t_{j \nu}:=g_{j \nu}\left(\underline{x}_{j}\right) / h_{j \nu}\left(\underline{x}_{j}\right)$ are defined locally as elements of $L$, and they are local representatives for $\Psi\left(t_{\nu}\right)$. We denote $\mathcal{B}_{j}=\left(t_{j 1}, \ldots, t_{j d}\right)$.

Now suppose that $\mathcal{B}_{d}:=\mathcal{B}$ has supplementary properties, as indicated in Theorem 1.4. More precisely, suppose that:

a) If $\kappa$ and thus $\lambda$ have positive characteristic $p>0$, then $\mathcal{B}$ is a separable transcendence basis of $K \mid \kappa$.

- Then the form $q_{\mathcal{B}}^{(p)}$ from loc.cit. does not represent 0 over $K$, thus over ${ }^{*} K$. Therefore, $\Psi\left(q_{\mathcal{B}}^{(p)}\right)=q_{\Psi(\mathcal{B})}^{(p)}$ does not represent 0 over ${ }^{*} L$. Equivalently, the form $q_{\mathcal{B}_{j}}^{(p)}$ does not represent 0 over $L$ locally. Again, by Theorem $1.4,(1)$, it then follows that $\mathcal{B}_{j}$ is locally a separable transcendence basis of $L \mid \lambda$.

b) If $\kappa$ is algebraically closed of characteristic zero, thus the same is true for $\lambda$, then the Pfister form $q_{\mathcal{B}}$ does not represent 0 over $K$, thus over ${ }^{*} K$.

- Then $\Psi\left(q_{\mathcal{B}}\right)=q_{\Psi(\mathcal{B})}$ does not represent 0 over ${ }^{*} L$. Equivalently, the Pfister form $q_{\mathcal{B}_{j}}$ does not locally represent 0 over $L$. Again, by Theorem $1.4,(2)$, it follows that $\mathcal{B}_{j}$ is locally a separable transcendence basis of $L \mid \lambda$.

c) If $\kappa$ be a number field, thus $\lambda$ is a number field too, then there exit $t_{d+1}, t_{d+2} \in \kappa$ such that the corresponding Pfister form $q_{\left(t_{1}, \ldots, t_{d+2}\right)}$ does not represent 0 over $K[\sqrt{-1}]$, thus over ${ }^{*} K[\sqrt{-1}]$. 
- Reasoning as above, and taking into account that $u_{d+1}=\Psi\left(t_{d+1}\right)$ and $u_{d+2}=\Psi\left(t_{d+2}\right)$ are in $\lambda$, it follows that the corresponding Pfister form $q_{\left(t_{j 1}, \ldots, t_{j d}, u_{d+1}, u_{d+2}\right)}$ does not represent 0 over $L[\sqrt{-1}]$. By Theorem $1.4,(3)$, it follows that $\mathcal{B}_{j}$ is locally a transcendence basis of $L \mid \lambda$.

From the discussion above we deduce that the subfield $L_{j}=\lambda\left(\underline{u}_{j}\right)$ of $L$ generated by $\underline{u}_{j}$ locally has transcendence degree $d$ over $\lambda$, thus $\underline{u}_{j}$ is a generic point of the variety $\mathcal{X}_{j} \rightarrow S_{j}$. Equivalently, we have obtained an approximation

$$
\varphi_{j}: \operatorname{Spec} L \rightarrow \mathcal{X}_{j} \stackrel{\text { can }}{\longrightarrow} \mathcal{X}
$$

where $\mathcal{X}_{j} \stackrel{\text { can }}{\longrightarrow} \mathcal{X}$ comes from the base change $\mathcal{X}_{j}=\mathcal{X} \times_{R} S_{j}$. By its construction, the approximation $\varphi_{j}$ is separable, provided $\mathcal{B}$ a separable transcendence base.

One concludes the proof of Theorem A and Theorem B by applying Theorem 2.1.

Remark. Under the hypothesis of assertion (2) of Theorem A and Theorem B, it follows that $\Psi\left(K^{*} \kappa\right)=L^{*} \lambda$.

\section{REFERENCES}

[B-T] Bass H. and TAte, J., The Milnor ring of a global field. Algebraic $K$-theory, II: "Classical" algebraic $K$-theory and connections with arithmetic (Proc. Conf., Seattle, Wash., Battelle Memorial Inst., 1972), pp. 349-446. LNM 342, Springer, Berlin 1973.

[B-S] Bell J.-L. and Slomson, A.-B, Models and ultra-products: an introduction, Amsterdam 1969.

[B] BLOCH, S., .

[BOU] Bourbaki, Algèbre commutative, Hermann Paris 1964.

[C-S] Cornell-Silverman, Introduction to arithmetic geometry, eds, .

[D1] Duret, J.-L., Sur la théorie élémentaire des corps de fonctions, J. Symbolic Logic 51 (1986), 948-956.

[D2] Équivalence élémentaire et isomorphisme des corps de courbe sur un corps algebriquement clos, J. Symbolic Logic 57 (1992), 808-923.

[D-S] VAn Den Dries, L. and Schmidt, K., Bounds in the theory of polynomial rings over fields. A non-standard approach, Invent. Math. 76 (1984), 77-91.

[F] Faltings, G., Endlichkeitssätze für abelsche Varietäten über Zahlkörpern, Invent. Math. 73 (1983), 349-366.

[Ei] Eisenbud, D., Commutative Algebra, GTM 150, Springer, 1999. 
[E-L] Elman, R. and Lam T.-Y., Pfister forms and K-theory of fields, J. Algebra 23 (1972), 181-213.

[H] Hartshorne, R. Algebraic Geometry, Springer Verlag, New York · Berlin . Heidelberg ... 1993 (sixth printing).

[I] Iitaka, Sh. Algebraic Geometry, Springer Verlag, New York · Berlin · Heidelberg 1982.

[J-R] JAcobs, B. and Rost, M., Degree four cohomological invariants for quadratic forms, Invent. Math. (1989), 552-570.

[Kh] Kahn, E., La conjecture de Milnor (d'après Voevodsky), Séminaire Bourbaki, Asterisque 245 (1997), 379-418.

[Ka] KAnI, E., Bounds on the number of non-rational subfields of a function field, Invent. Math. 85 (1986), 185-198.

[Ko] Kосн, H., Die Galoissche Theorie der $p$-Erweiterungen, Math. Monographien 10, Berlin 1970.

[M-S1] Merkurjev, A.-A. and Suslin, A., Merkurjev, A. S., K2 of a field, and the Brauer group, Sov.Math. Doklady 24 (1981), 546-551.

[M-S2] Merkurjev, A. S. and Suslin A. A., K-cohomology of Brauer-Severi varieties and the norm residue homomorphism, Math. USSR Isz. ??? (1983), 307-340.

[M1] Milnor, J., Algebraic K-theory and quadratic forms, Invent. Math. 9 (1970), $318-344$.

[M2] Introduction to algebraic K-theory, Ann. Math. Studies 72, Princeton Univ. Press 1971.

[Pa $]$ Parshin, A. N., Finiteness Theorems and Hyperbolic Manifolds, in The Grothendieck Festschrift III, ed P. Cartier et all, PM Series Vol 88, Birkhäuser Boston Basel Berlin 1990.

[Pi] Pierce, D., Function fields and elementary equivalence, Bull. London Math. Soc.31 (1999), 431-440.

[P] Pop, F., On Grothendieck's conjecture of anabelian geometry, Ann of Math. 138 (1994), 145-182.

[R-R] Robinson, A. and Roquette, P., On the finiteness of Siegel and Mahler concerning Diophantine equations, J. Number Theory 7 (1975), 121-176.

[Q] Quillen, D., Higher algebraic K-Theory I, in: Springer LNM 341, 85-147.

[R] Rost, M., see References in [Kh] above.

[S] Serre, J.-P., Cohomologie Galoisienne, LNM 5, Springer 1965.

[S-V] Suslin, A. A. and Vojevodsky, V., Bloch-Kato conjecture and motivic cohomology with finite coefficients, preprint (1995).

[V] Vojevodsky, V., see References in [Kh] above.

[Z-S] Zariski, O. and Samuel, P., Commutative Algebra, Van Nostrand, Princeton 1958 and 1960. 